John P. Boyd*

\title{
Revisiting the Charney Baroclinic Instability Problem and Point-jet Barotropic Instability Problem, Part II: Matched Asymptotic Expansions \& Overreflection Without Delta-Functions
}

https://doi.org/10.1515/mcwf-2018-0004

Received August 28, 2018; accepted December 18, 2018

\begin{abstract}
Baroclinic instability generates the cyclones and anticyclones of midlatitude weather. Charney developed the first effective theory for the infancy of this cyclogenesis in 1947. His linear eigenproblem is analytically solvable by confluent hypergeometric functions. It is also, with extension of the domain of the coordinate from $[0, \infty]$ to $[-\infty, \infty]$ by reflection about the origin, the point-jet model of barotropic instability, important for tropical cyclogenesis. (Note that the coordinate is height $z$ in the Charney model, but latitude $y$ for the point-jet bartropic instability. It is a great simplification that the Charney and point-jet instability problems are mathematically identical, but it also is confusing that the mathematical analysis in $y$ also applies to the Charney problem with the substitution of $z$ for $y$.)

Unfortunately, the theory is full of distributions like the Dirac delta-function and the reflected Charney eigenfunction has a discontinuous first derivative at $y=0$. Here we regularize the Charney problem by replacing a linear mean current, $U=|y|$, by either $U=\epsilon \log (\cosh (y / \epsilon))$ or $U=\epsilon y \operatorname{erf}(y / \epsilon)$, followed by matched asymptotic perturbation expansions in powers of the small regularization parameter $\epsilon$. The series is carried to third order because the lowest nonzero correction to the phase speed is $O\left(\epsilon^{2}\right)$ and this correction is determined simultaneously with the third order approximation to the eigenfunction. The result is both an explicit, analytic regularization of a problem important in atmospheric and ocean dynamics, but also a good school problem because the series is explicit with nothing worse than polylogarithms and confluent hypergeometric functions. The primary meteorological conclusion is that the delta functions in the Charney problem are harmless as demonstrated both by third order perturbation theory and by spectrally-accurate numerical solutions. The physics of the regularized Charney problem is not significantly changed from that of the original Charney problem.
\end{abstract}

Keywords: baroclinic instability;singular perturbation; barotropic instability

MSC: 76E20; 86A10; 35B25; 33C15

"The argument which demands no horizontal variation of potential temperature at the boundary but a finite gradient an infinitesimal distance above I find most disquieting. It is, for example, difficult to escape the conclusion that since $\mathrm{N}^{\star \star} 2$ is continuous) there must be large areas of static instability in this boundary layer. In addition such a velocity profile is itself in a sense "critical" for (as reported in Green (1960), equation 11) an additional baroclinic wave may exist if the horizontal temperature gradient changes sign a finite,

^Corresponding Author: John P. Boyd: Department of Climate \& Space Sciences and Engineering, University of Michigan, 2455 Hayward Avenue, Ann Arbor MI 48109, E-mail: jpboyd@umich.edu 
possibly small, height above the boundary."

John S. A. Green, in comments on Francis P. Bretherton's papers "Critical layer instability in baroclinic flows" and "Baroclinic instability and the short wavelength cutoff in terms of potential vorticity". Published in the discussion section of the Quarterly Journal Of the Royal Meteorological Society, page 552 (1967) [12].

\section{Introduction}

In recent years, two quite different conceptual models have competed to explain barotropic and baroclinic instability. Wave Overreflection Theory, usually abbreviated as OR, depends upon a configuration of turning points and critical levels that allow waves to grow as normal modes like light bouncing back and forth between the silvered ends of a laser. Richard Lindzen and his students were the most enthusiastic contributors to OR theory, mostly in the 80 's $[1,19,21-25,32,33,33,34,36]$. The very different Counter-propagating Rossby Wave (CRW) paradigm depends on a resonant interaction between a pair of Rossby waves propagating in different directions [7, 13-17, 26, 27]. The two conceptual perspectives are not mutually exclusive, but are quite dramatically different. The Orr mechanism plays a significant role in both theories of exponentially-growing instability [19], but in its original form $[2,10,28]$ is a theory of the transient growth of the continuous spectrum, followed by asymptotic decay for large times. It is important to understand these mechanisms because baroclinic instability generates the continent-sized storms that constitute most of the large-scale weather in the middle latitudes; barotropic instability of the East African jet initiates most Atlantic hurricanes.

The prototype of all baroclinic instability models is the "Charney problem", described in detail below, which is an eigenproblem for waves linearized about a mean flow which varies linearly with height $[9,11,18]$. Schoeberl and Lindzen showed that a barotropic flow with linear mean shear in latitude is mathematically isomorphic to the Charney problem of baroclinic instability if the intervals of linear shear are joined by an infinitesimal layer in which the relative vorticity is a Dirac delta function and the shear of the mean current $U$ is discontinuous. Although the underlying physics of baroclinic and barotropic instability is quite different, the Charney problem is a key to both.

This paper is the second in a multipart series, following [6], which hopes to create a better synthesis of the OR, CRW and Orr paradigms by revisiting and extending simple, classic problems with modern analytical and computational technologies.

One flaw in current theories of barotropic and baroclinic instability is that both OR and CRW are inordinately fond of delta functions. Here, we use the method of matched asymptotic expansions to show that the Charney problem can be regularized to delete the delta functions and also, it turns out, show that the infinitesimal layer results of earlier work are correct in the limit that the layer depth goes to zero.

Our tool is the method of matched asymptotic expansions. The Charney eigenrelation falls out at first order. Nevertheless, we have extended the expansion to third order for three reasons. First, some perturbation series have logarithms of the perturbation parameter appear at higher order, or may break down entirely, as common in the method of strained coordinates. The regularity of the third order approximation strongly suggests that nothing unconventional happens at any order. Second, the lowest non trivial correction to the phase speed $c$ is at second order, but is determined in the calculation of the third order eigenfunction. Third, this is a good pedagogical example because it is a rare problem where one can explicitly go to third order while encountering only polylogarithms and confluent hypergeometric functions.

We explicitly employ two different regularized mean current profiles $U(y)$. Both are smoothed versions of the function $U(y)=|y|$ and are chosen for smoothness and simplicity rather than observational curve-fitting. Both profiles give solutions to the wave equation which are analytic for all real $y$. The $\log (\cosh )$ function has singularities in the complex plane and asymptotes to $|y|$ for large $|y|$ as $U \sim|y|+O(\exp (-2|y|)$. The linear-erf function is an entire function that asymptotes to $|y|$ for large $|y|$ as $U \sim|y|+O\left(\exp \left(-y^{2}\right)\right)$.

Regularization has been previously considered by Bretherton [8] and Lindzen and Tung [24]. Without performing either numerical or perturbative calculations these authors noted that a delta function in potential 
vorticity can be removed by smoothing the linear variation of the mean flow so that the shear at the ground is zero. Lindzen, Farrell and Tung [21] number-crunched to show that it is possible to completely eliminate the instability by curving the wind profile so that $U_{z}$ is zero at the ground. They also did not derive any perturbative expansions.

\section{The Charney Model of Baroclinic Instability}

The vertical structure function $u(z)$ wherer $u$ is the streamfunction and not the zonal velocity, is

$$
\begin{array}{r}
u_{z z}+\left\{\frac{r}{z-c}-\frac{1}{4}\right\} u=0, \quad z \in[0, \infty] \\
c u_{z}+u=0 \text { at } z=0, \quad u(z) \rightarrow 0 \text { as } z \rightarrow \infty
\end{array}
$$

The complete streamfunction is the product of a trigonometric function in latitude multiplied by exp $(i k(x-c t))$ where $c$ is the phase speed and $k$ is the zonal wavenumber. The parameter $r$ is the nondimensionalized beta parameter (the latitudinal derivative of the Coriolis parameter).

This equation arises through separation-of-variables from a special case of the general equation for a wave, linearized about a mean zonal wind $U(y, z)$, in the quasi-geostrophic approximation, which is given as (6.70) and (13.42) of the textbooks by Vallis [35] and Lindzen [20], respectively. Good reviews of quasigeostrophic instability can also be found in the text by Pedlosky [29] and the review by Pierrehumbert and Swanson [31]. The history of Charney's own work is described in [30].

Kuo gave an alternative form which is more convenient for analysis [18]:

$$
\begin{aligned}
u_{\eta \eta}+\left\{\frac{r}{\eta}-\frac{1}{4}\right\} u & =0 \\
\eta u_{\eta}-u & =0 \text { at } \eta=\eta_{0}, \quad u(\eta) \rightarrow 0 \text { as } \eta \rightarrow \infty
\end{aligned}
$$

where $\eta_{0}$ is the unknown eigenparameter where the solution which is bounded as $\eta \rightarrow \infty$ satisfies the boundary condition. The coordinates are related by

$$
\eta \equiv z-c \quad \leftrightarrow \quad z=\eta+c
$$

where $c$ is the complex-valued phase speed. Note that at the ground $\eta=-c=\eta_{0}$, which translates the boundary condition of the first form into the boundary condition of the second form.

Instabilities occur for all $r>0$ except when $r$ is an integer, but the primary branch, the fastest-growing waves, is on $r \in[0,1]$, so we shall concentrate on this range. The eigenvalues for this parametric interval is shown in Fig. 1.

The solution to the Kuo form which decays exponentially with increasing $z$ (or $\eta$ ) so as to be bounded as $|z| \rightarrow \infty$ is the one-parameter Whittaker function. Denoting the confluent hypergeometric by $\mathrm{M}(a, b ; z)$ and $\mathrm{U}(a, b ; z)$,

$$
W_{r, 1 / 2}(\eta)=\exp (-\eta / 2) \eta U(1-r, 2, \eta)
$$

The second linearly independent solution is

$$
M_{r, 1 / 2}(\eta)=\exp (-\eta / 2) \eta M(1-r, 2, \eta)
$$

The M-function is analytic at $\eta=0$, which is the location of the pole in the coefficient of the differential equation. This is in contrast to the W-function, which is logarithmically singular at $\eta=0$. The M-function grows exponentially as $z \rightarrow \infty$ and so must be rejected as a solution in its own right, but ironically is still needed because the coefficient of the logarithm in the Whittaker W-function is none other than a constant times the Whittaker M-function! 
Note that $\eta_{0}$ is always the negative of the corresponding phase speed $c$. The power series in the Whittaker functions have infinite radii of convergence:

$$
\begin{aligned}
M(1-r, 2, \eta)=\sum_{m=0}^{\infty} \frac{(1-r)_{m}}{(2)_{m}} \frac{\eta^{m}}{m !} \\
\Gamma(1-r) U(1-r, 2, \eta)=\frac{1}{\eta}-r M(1-r, 2, \eta)\{\log |\eta|-\alpha \pi i\} \\
-r \sum_{m=0}^{\infty} \frac{(1-r)_{m}}{(2)_{m}} \frac{\eta^{m}}{m !}\{\psi(1-r-m)-\psi(1+m)-\psi(2+m)\} \\
(x)_{m} \equiv x(x+1) \ldots(x+m-1)
\end{aligned}
$$

where $\psi$ is the digamma function.

The branch cut is in the upper $\eta$-plane implying that $\alpha=1$ in (9). The logarithmic part of the U-function function is

$$
\begin{aligned}
U^{\log }(1-r, 2, \eta) & =-\frac{r}{\Gamma(1-r)} \frac{1}{\eta} M(1-r, 2, \eta) \log (\eta) \\
& =-\frac{r}{\Gamma(1-r)} \frac{1}{\eta} M(1-r, 2, \eta)\{\log |\eta|-\pi i\}
\end{aligned}
$$

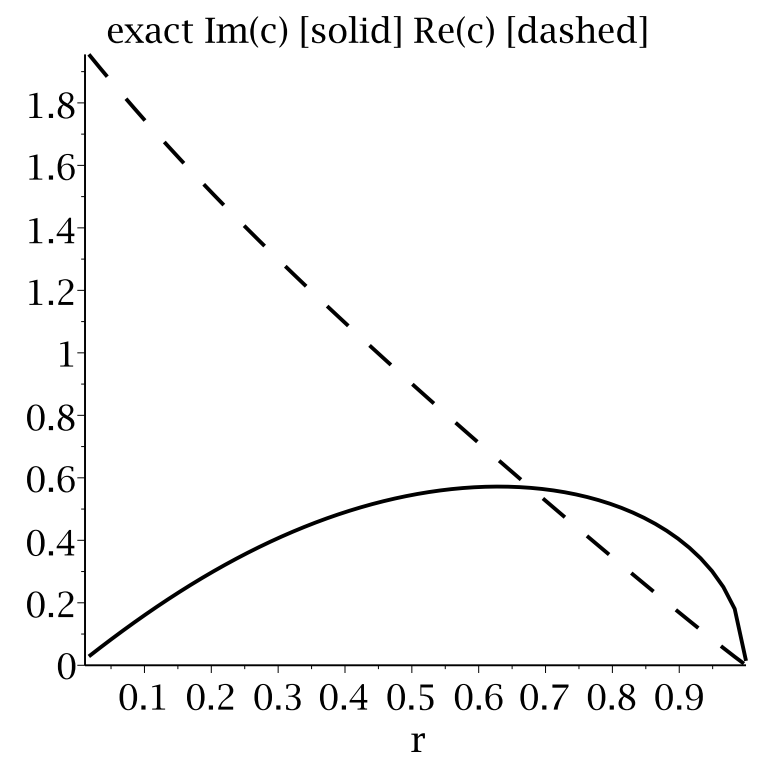

Figure 1: Real and imaginary parts for the phase speed $c$ in the Charney problem.

\section{The Point-Jet Model of Barotropic Instability}

Suppose that the mean current is

$$
U(y)=|y|, \quad y \in[-\infty, \infty] \quad \text { ["Point-Jet”] }
$$


where $y$ is latitude. Lindzen, Rosenthal and Farrell showed that the barotropic instability of the "point-jet" in an unbounded flow is mathematically identical with Charney's eigenproblem [23]. (The Charney problem is defined on a semi-infinite domain; strictly speaking, therefore, the solution to the barotropic point jet instability is the Charney eigenfunction $\psi(y), y=[0, \infty]$ plus its reflection about $y=0, \psi(-y)=\psi(y) \forall y)$. The Charney problem and the point jet barotropic instability are not physically isomorphic because baroclinic instability is a transfer of available potential energy whereas barotropic instability is an exchange of kinetic energy.

Nevertheless, this mathematical equivalence greatly strengthens the case for Lindzen's sweeping view of overreflection as the primary mechanism of both barotropic and baroclinic instability.

Point jet (solid) \& Regularized Mean Wind (dashed)

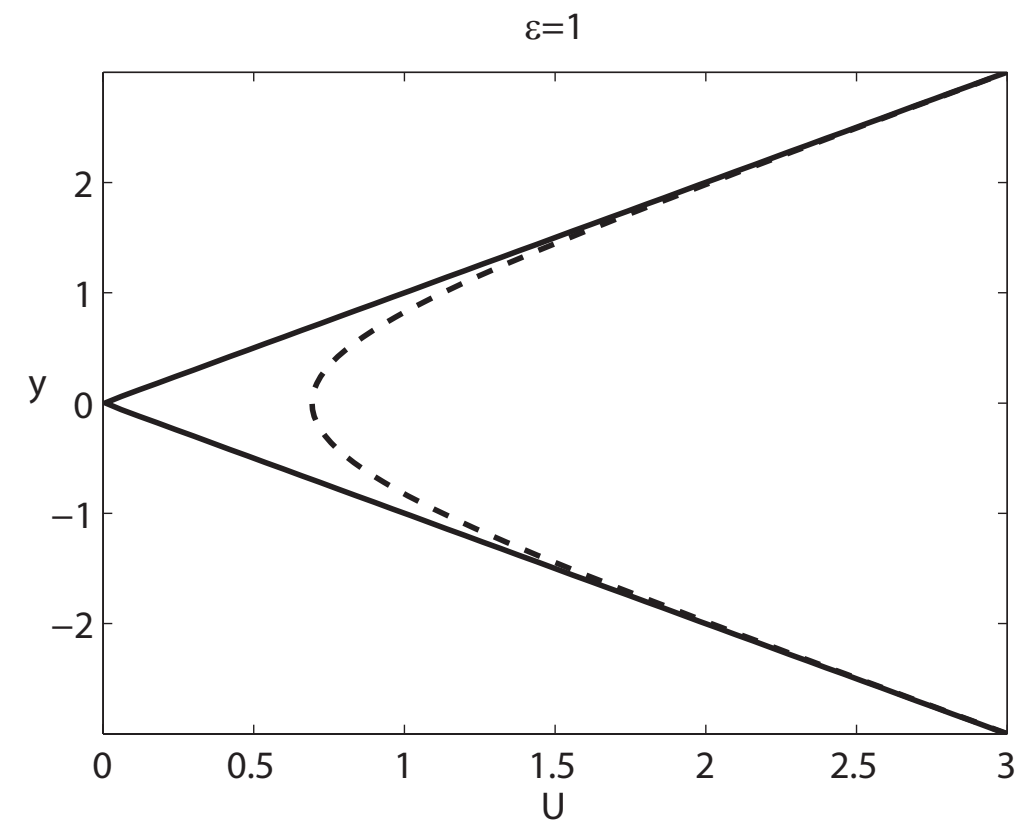

Figure 2: Solid: The point-jet mean current $U(y)=|y|$. Dashed; the regularized mean flow $U(y)=\epsilon[\log (\cosh (y / \epsilon))-\log (2)]$ for $\epsilon=1$.

\section{Regularizing the Point-Jet and Charney problems}

The mean vorticity of the point-jet is a Dirac delta function. It is obviously desirable to remove the singularity. (Students, I have found in forty-one years of teaching, are particularly unhappy with infinitesimally thin, infinitely large quantities.) To regularize the point jet, we replace the piecewise-linear mean wind by a smooth function with the following properties where $\epsilon$ is a small positive parameter:

1.

$$
U(y) \sim y+\text { exponentially fast in }(y / \epsilon)), \quad|y| \rightarrow \infty
$$

2.

$$
U(-y)=U(y) \forall y \text { [Symmetry with respect to the origin] }
$$

Two specific instances of this class are the " $\log (\cosh ) "$ jet defined by

$$
\begin{aligned}
U(y ; \epsilon) & =\epsilon\{\log (\cosh (y / \epsilon))+\log (2)\} \\
& \sim|y|, \quad|y| \gg \epsilon
\end{aligned}
$$


and the "linear-erf" jet defined by

$$
\begin{aligned}
U(y ; \epsilon) & =\epsilon\{(y / \epsilon)) \operatorname{erf}(y / \epsilon)\} \\
& \sim|y|, \quad|y| \gg \epsilon
\end{aligned}
$$

Fig. 2 compares the piecewise linear mean flow with the regularized current that will replace it. In the limit that the parameter $\epsilon$ tends to zero, the $\log (\cosh )$ profile reverts to the point jet. For all nonzero $\epsilon$, the infinite vorticity is smeared out into a layer of thickness $O(\epsilon)$.

There are many functions that share the same limiting and approximation properties. Our choice of the logarithm of the hyperbolic cosine and the linear-erf were motivated by the fact that the derivatives of both are known explicitly, which means that the coefficients of the regularized differential equation will be explicit elementary functions also; in addition, all the integrals are known analytically as cataloged in Table 1 and Table 2, which is very helpful in applying perturbation theory. A further advantage is that both functions tends exponentially fast to the point-jet as $|y| \rightarrow \infty$. When $\epsilon$ is small, the differential equation close to Charney's except in a region of width $O(\epsilon)$ around the ground (in the baroclinic instability interpretation) or the discontinuity at the maximum of the point-jet (in the barotropic instability application).

Our chosen analytical method is to apply matched asymptotic expansions in powers of $\epsilon$. The narrow regularization interval around the origin is the "inner region" in the parlance of perturbation theory; the rest of the domain is the "outer region" where the approximation is a confluent hypergeometric function to all orders, just as in the original Charney problem.

We will treat general $U(y)$ first and then specialize to the " $\log (\cosh )$ " and "linear-erf" jets later.

The regularized problem is, introducing the inner coordinate $Y \equiv y / \epsilon$,

$$
u_{y y}+\left\{\frac{r-\epsilon^{-1} \tilde{U}_{Y Y}(y / \epsilon)}{\epsilon \tilde{U}(y / \epsilon)-c}-1 / 4\right\} u=0, \quad u_{y}(0)=0, \quad u( \pm \infty)=0
$$

where $U(y / \epsilon ; \epsilon) \equiv \epsilon \tilde{U}(y / \epsilon)$. Since the ODE is symmetric about $Y=0$, it is easy to show by the parity analysis of Chapter 8 of [4] that all the eigenmodes are either symmetric or antisymmetric with respect to the origin. We shall focus on symmetric modes only as consistent with the boundary condition $u_{y}(0)=0$.

Antisymmetric instability modes are also possible. However, these will not be analyzed here since the symmetric modes suffice to analyze OR and CRW concepts.

The eigenfunctions need to be normalized; we shall impose

$$
u(0)=1
$$

which is a condition that must be satisfied by the inner solution.

\section{Matched Asymptotic Expansions}

The key assumption is

$$
\epsilon \ll 1
$$

The regularized eigenfunction will be well-approximated by the Whittaker function except in a thin boundary layer around $y=0$, the "inner region".

\subsection{Outer Solution}

By assumption, the mean wind $\epsilon U(y / \epsilon)$ tends exponentially fast to the point-jet as $|y| \rightarrow \infty$.This implies that there will be no corrections in powers of $\epsilon$ to the coefficients of the differential equation in the outer region. Therefore, the outer equation to all orders is

$$
u_{y y}+\left\{\frac{r}{y-c}-1 / 4\right\} u=0
$$


However, note that the phase speed $c$ has $O\left(\epsilon^{2}\right)$ corrections. The outer solution is

$$
u^{\text {outer }}(y)=\mathfrak{A} \frac{W_{r, 1 / 2}(y-c)}{W_{r, 1 / 2}\left(-c_{0}\right)}
$$

where the denominator $W_{r, 1 / 2}\left(-c_{0}\right)$ is convenient because then $\lim _{\epsilon \rightarrow 0} u^{\text {outer }}(y=0)=\mathfrak{A}$. Both the amplitude $\mathfrak{A}$ and $c$ are functions of $\epsilon$ and therefore must be formally expanded as

$$
\begin{aligned}
c & \sim c_{0}+c_{1} \epsilon+c_{2} \epsilon^{2}+c_{3} \epsilon^{3}+\ldots \\
\mathfrak{A} & \sim P_{0}+P_{1} \epsilon+P_{2} \epsilon^{2}+P_{3} \epsilon^{3}+\ldots
\end{aligned}
$$

It is convenient to define

$$
w(y ; r)=W_{r, 1 / 2}(y)
$$

where $W_{r, \kappa}(z)$ is the standard Whittaker function. We shall usually suppress the parameter $r$ for notational simplicity. Furthermore, we shall adopt the convention that whenever $w$ or one of its $y$-derivatives is written without arguments, the implied argument is $-c_{0}$, corresponding to $y=0$. Thus,

$$
\begin{aligned}
w & \leftrightarrow w\left(-c_{0}\right)=W_{r, 1 / 2}\left(-c_{0}\right) \\
w_{y} & \leftrightarrow w_{y}\left(-c_{0}\right)
\end{aligned}
$$

and so on.

\subsection{Inner Approximation}

\subsubsection{The inner problem}

The inner coordinate $Y$ is defined by $y=\epsilon Y$.

The inner equation is

$$
u_{Y Y}+\left\{\frac{r \epsilon^{2}-\epsilon U_{Y Y}(Y)}{\epsilon U(Y)-c}-(1 / 4) \epsilon^{2}\right\} u=0
$$

and the boundary conditions are $u_{Y}(0)=0$ (to impose symmetry), and $u(0)=1$ (arbitrary normalization of the eigenfunction).

$$
\begin{gathered}
u(Y)=u_{0}^{i}(Y)+u_{1}^{i}(Y) \epsilon+u_{2}^{i}(Y) \epsilon^{2}+\ldots \\
c=c_{0}+c_{1} \epsilon+c_{2} \epsilon^{2}+\ldots
\end{gathered}
$$

\subsubsection{The inner solution}

At lowest order, we have, after imposing the boundary conditions, $u_{0}^{i}(0)=1$,

$$
u_{0, Y Y}^{i}=0, \quad \rightarrow u_{0}^{i}(Y)=1
$$

The first-order equation is

$$
u_{1, Y Y}^{i}=-\frac{1}{c_{0}} U_{Y Y}(Y), \quad u_{1}(0)=0, u_{1, Y}(0)=0
$$

which is solved by

$$
u_{1}^{i}(Y)=-\frac{1}{c_{0}}(U(Y)-U(0))
$$


The second order equation and second-order solution are

$$
\begin{gathered}
u_{2, Y Y}^{i}=\frac{1}{4}+\frac{r}{c_{0}}+\frac{c_{1} / c_{0}}{c_{0}} U_{Y Y}(Y) \\
u_{2}^{i}=\left(\frac{1}{8}+\frac{r}{2 c_{0}}\right) Y^{2}+\left\{\frac{c_{1}-U(0)}{c_{0}^{2}}\right\}\{U(Y)-U(0)\}
\end{gathered}
$$

At third order,

$$
u_{3, Y Y}^{i}=\frac{r U(0)}{c_{0}^{2}}+\left\{\frac{c_{2}}{c_{0}^{2}}-\frac{U(0)^{2}}{c_{0}^{3}}\right\} U_{Y Y}(Y)-\frac{1}{4 c_{0}}(U(Y)-U(0))-\frac{1}{c_{0}}\left\{\frac{1}{8}+\frac{r}{2 c_{0}}\right\} Y^{2} U_{Y Y}
$$

It is convenient to define $V(Y)$ as the iterated integral of the mean wind, more precisely,

$$
\begin{gathered}
V_{Y Y}=U(Y), \quad V(0)=V_{Y}(0)=0 \\
u_{3}^{i}=\frac{r U(0)}{2 c_{0}^{2}} Y^{2}+\left\{\frac{c_{2}}{c_{0}^{2}}-\frac{U(0)^{2}}{c_{0}^{3}}\right\}(U(Y)-U(0))-\frac{1}{4 c_{0}}\left(V(Y)-(U(0) / 2) Y^{2}\right) \\
-\frac{1}{c_{0}}\left\{\frac{1}{8}+\frac{r}{2 c_{0}}\right\}\left\{Y^{2} U(Y)-4 Y V_{Y}+6 V(Y)\right\}
\end{gathered}
$$

We need the following to derive the outer limit of the inner solution.

$$
\begin{aligned}
U(Y) & \sim Y \\
V_{Y} & \sim v_{1}+Y^{2} / 2 \\
V(Y) & \sim v_{0}+v_{1} Y+Y^{3} / 6
\end{aligned}
$$

where $V(Y)$ is the iterated integral of the mean wind defined by (37). Note that $V_{Y}$ cannot include an asymptotic term that is linear in $Y$ because this would imply that $U(Y)=\left(V_{Y}\right)_{Y}$ would asymptote to a constant plus a linear term, contrary to our assumptions on $U(Y)$. Similarly, $V_{Y}$ cannot include a constant in its asymptotic form unless $V(Y)$ has a term linear in $Y . V(Y)$ cannot include a quadratic term because this would force a constant in $U(Y)$. The outer limit of the inner approximation is

$$
\begin{aligned}
u_{0}^{i, \text { outer }}= & 1 \\
u_{1}^{i, \text { outer }}= & -\frac{1}{c_{0}} Y+\frac{1}{c_{0}} U(0) \\
u_{2}^{i, \text { outer }}= & \left(\frac{1}{8}+\frac{r}{2 c_{0}}\right) Y^{2}+\left\{\frac{c_{1}-U(0)}{c_{0}^{2}}\right\} Y-\left\{\frac{c_{1}-U(0)}{c_{0}^{2}}\right\} U(0) \\
u_{3}^{i, \text { outer }}= & -\frac{1}{24} \frac{Y^{3}}{c_{0}}+\left(\frac{r U(0)}{2 c_{0}^{2}}+\frac{U(0)}{8 c_{0}}\right) Y^{2} \\
& +\left(-2\left(\frac{1}{8}+\frac{r}{2 c_{0}}\right) \frac{v_{1}}{c_{0}}+\frac{c_{2}}{c_{0}^{2}}-\frac{(U(0))^{2}}{c_{0}^{3}}-\frac{v_{1}}{4 c_{0}}\right) Y \\
& -\left(\frac{c_{2}}{c_{0}^{2}}-\frac{(U(0))^{2}}{c_{0}^{3}}\right) U(0)-6\left(\frac{1}{8}+\frac{r}{2 c_{0}}\right) \frac{v_{0}}{c_{0}}-\frac{v_{O}}{4 c_{0}}
\end{aligned}
$$

The inner limit of the outer solution is

$$
\begin{aligned}
u^{o, \text { inner }} \sim & P_{0}+\epsilon\left\{P_{1}+P_{0}\left(-\frac{w_{y}}{w} c_{1}+\frac{w_{y}}{w} Y\right)\right\} \\
& +\epsilon^{2}\left\{P_{2}+P_{1}\left(-\frac{w_{y}}{w} c_{1}+\frac{w_{y}}{w} Y\right)\right. \\
+P_{0} & \left.\left(\left[\frac{1}{2} \frac{w_{y y}}{w} c_{1}^{2}-\frac{w_{y}}{w} c_{2}+\right]-\frac{w_{y y}}{w} c_{1} Y+\frac{1}{2} \frac{w_{y y}}{w} Y^{2}\right)+P_{1}\right\}+\ldots
\end{aligned}
$$




\section{Term-by-Term Matching}

\subsection{Overview of Matching the Inner \& Outer Solutions}

When $Y \gg 1 \leftrightarrow y \gg 1 / \epsilon$, the exponentially decaying terms are negligible and the inner approximation, truncated at any finite order $m$ in $\epsilon$, is a polynomial in $Y$. If $y<<1$, the outer approximation is accurately approximated by its power series in $y$. There is thus an overlap region, $\epsilon \ll y \ll 1$, where both approximations are simultaneously valid. Note that in the overlap region, the exponentials have decayed so that the inner approximation is just a polynomial in $Y$ and $\epsilon$. Simultaneous validity requires that the coefficients of the power series from the outer approximation and the polynomials that are the inner approximation must match, term by term, so that the approximations agree simultaneously not merely at points, but on an interval.

The inner limit of the outer solution is just a bivariate power series in $\epsilon$ and $y$ with coefficients we shall denote by $p_{j k}$ :

$$
\text { Inner limit }\left(u_{\text {outer }}\right) \sim \sum_{j=0} \sum_{k=0} p_{j k} \epsilon^{j} y^{k}
$$

Before conversion to the outer variable $y$, the inner solution is the bivariate power series with coefficients denoted by $q_{j k}$ :

$$
\text { Outer limit }\left(u_{\text {inner }}\right) \sim \sum_{j=0} \sum_{k=0} q_{j k} \epsilon^{j} Y^{k}
$$

Recall that $y=\epsilon Y \quad \leftrightarrow \quad Y=y / \epsilon$. Then

$$
\operatorname{OuterLimit}\left(u_{\text {inner }}\right) \sim \sum_{j=0} \sum_{k=0}^{j} q_{j k} \epsilon^{j-k} y^{k}
$$

It follows that in the overlap region at $O\left(\epsilon^{m}\right)$,

$$
\sum_{k=0}^{m} p_{m-k, k} y^{k}=\sum_{k=0} q_{m k} y^{k}
$$

which yields the matching condition

$$
p_{m-k, k}=q_{m k}, \quad k=0,1, \ldots m
$$

If $k=m+1$, matching requires $p_{-1, k}$, a coefficient of $1 / \epsilon$ in the outer solution. If this coefficient is zero, then the inner solution must be a polynomial in $Y$ of at most degree $m$, or in other words $q_{m k}$ is a triangular array in the sense that

$$
q_{m, k}=0, \quad \forall k>m, \quad m=0,1, \ldots
$$

The $p_{j k}$ up to third order are explicitly

$$
\begin{gathered}
O(1):\left\{\begin{array}{l}
p_{00}=P_{0} \\
p_{01}=\frac{w_{y}}{w}=-\frac{1}{c_{0}} \\
p_{02}=\frac{1}{2} \frac{w_{y y}}{w}=\frac{r}{2 c_{0}}+\frac{1}{8} \\
p_{03}=\frac{1}{6} \frac{w_{y y y}}{w_{y}}
\end{array}\right. \\
O(\epsilon):\left\{\begin{array}{l}
p_{10}=P_{1}-c_{1} \frac{w_{y}}{w} \\
p_{11}=P_{1} \frac{w_{y}}{w}-c_{1} \frac{w_{y y}}{w} \\
p_{12}=P_{1} \frac{1}{2} \frac{w_{y y}}{w}-c_{1} \frac{1}{2} \frac{w_{y y y}}{w} \\
p_{13}=P_{1} \frac{1}{6} \frac{w_{y y y}}{w_{y}}-c_{1} \frac{1}{6} \frac{w_{y y y y}}{w}
\end{array}\right.
\end{gathered}
$$




$$
\begin{aligned}
& O\left(\epsilon^{2}\right):\left\{\begin{array}{l}
p_{20}=P_{2}-P_{1} c_{1} \frac{w_{y}}{w}+\frac{1}{2} c_{1}^{2} \frac{w_{y y}}{w}-c_{2} \frac{w_{y}}{w} \\
p_{21}=P_{2} \frac{w_{y}}{w}-c_{1} P_{1} \frac{w_{y y}}{w}+c_{1}^{2} \frac{1}{2} \frac{w_{y y y}}{w}-c_{2} \frac{w_{y y}}{w} \\
p_{22}=P_{2} \frac{1}{2} \frac{w_{y y}}{w}-P_{1} c_{1} \frac{1}{2} \frac{w_{y y y}}{w}+c_{1}^{2} \frac{1}{4} \frac{w_{y y y y}}{w}-c_{2} \frac{1}{2} \frac{w_{y y y}}{w} \\
p_{23}=P_{2} \frac{1}{6} \frac{w_{y y y}}{w_{y}}-P_{1} c_{1} \frac{1}{6} \frac{w_{y y y y}}{w}+c_{1}^{2} \frac{1}{12} \frac{w_{5 y}}{w}-c_{2} \frac{1}{6} \frac{w_{y y y}}{w}
\end{array}\right. \\
& O\left(\epsilon^{3}\right):\left\{\begin{array}{l}
p_{30}=P_{3}-P_{2} c_{1} \frac{w_{y}}{w}+P_{1} \frac{1}{2} c_{1}^{2} \frac{w_{y y}}{w}-P_{1} c_{2} \frac{w_{y}}{w}-\frac{1}{6} c_{1}^{3} \frac{w_{y y y}}{w}+c_{1} c_{2} \frac{w_{y y}}{w}-c_{3} \frac{w_{y}}{w} \\
p_{31}=P_{3} \frac{w_{y}}{w}-P_{2} c_{1} \frac{w_{y y}}{w}+P_{1} \frac{1}{2} c_{1}^{2} \frac{w_{y y y}}{w}-P_{1} c_{2} \frac{w_{y y}}{w}-\frac{1}{6} c_{1}^{3} \frac{w_{y y y y}}{w}+c_{1} c_{2} \frac{w_{y y y}}{w}-c_{3} \frac{w_{y y}}{w} \\
p_{32}=P_{3} \frac{1}{2} \frac{w_{y y}}{w}-P_{2} \frac{1}{2} c_{1} \frac{w_{y y y}}{w}+P_{1} \frac{1}{4} c_{1}^{2} \frac{w_{4 y}}{w}-P_{1} c_{2} \frac{1}{2} \frac{w_{y y y}}{w}-\frac{1}{12} c_{1}^{3} \frac{w_{5 y}}{w}+c_{1} c_{2} \frac{1}{2} \frac{w_{4 y}}{w}-c_{3} \frac{1}{2} \frac{w_{y y y}}{w} \\
p_{33}=P_{3} \frac{1}{6} \frac{w_{y y y}}{w}-P_{2} \frac{1}{6} c_{1} \frac{w_{4 y}}{w}+P_{1} \frac{1}{12} c_{1}^{2} \frac{w_{5 y}}{w}-P_{1} c_{2} \frac{1}{6} \frac{w_{4 y}}{w}-\frac{1}{36} c_{1}^{3} \frac{w_{6 y}}{w}+c_{1} c_{2} \frac{1}{6} \frac{w_{5 y}}{w}-c_{3} \frac{1}{6} \frac{w_{4 y}}{w}
\end{array}\right.
\end{aligned}
$$

The pertinent coefficients of the bivariate power series for the inner solution are the following:

$$
\begin{aligned}
& O(1): \quad q_{00}=1 \\
& O(\epsilon):\left\{\begin{array}{l}
q_{10}=-\frac{1}{c_{0}} U(0) \\
q_{11}=-\frac{1}{c_{0}}
\end{array}\right. \\
& O\left(\epsilon^{2}\right):\left\{\begin{array}{l}
q_{20}=-\left\{\frac{c_{1}-U(0)}{c_{0}^{2}}\right\} U(0) \\
q_{21}=\frac{c_{1}-U(0)}{c_{0}^{2}} \\
q_{22}=\frac{1}{8}+\frac{r}{2 c_{0}}
\end{array}\right. \\
& O\left(\epsilon^{3}\right):\left\{\begin{array}{l}
q_{30}=-\frac{v_{0}}{c_{0}}-3 r \frac{v_{0}}{c_{0}^{2}}-\frac{c_{2}}{c_{0}^{2}} U(0)+\frac{U(0)^{3}}{c_{0}^{3}} \\
q_{3,1}=-\frac{v_{1}}{2 c_{0}}-r \frac{v_{1}}{c_{0}^{2}}+\frac{c_{2}}{c_{0}^{2}}-\frac{(U(0))^{2}}{c_{0}^{3}} \\
q_{3,2}=U(0)\left(\frac{1}{8 c_{0}}+\frac{r}{2 c_{0}^{2}}\right) \\
q_{3,3}=-\frac{1}{24 c_{0}}
\end{array}\right.
\end{aligned}
$$

\subsection{Zeroth Order Matching}

The match of lowest order terms $p_{00}=q_{00}$ gives, recalling that $p_{00}=P_{0}$ and $q_{00}=1$, requires that

$$
P_{0}=1
$$

where $P_{0}$ is the leading coefficient in the amplitude constnat that appears in the outer approximation, $\mathfrak{A}(\epsilon)=$ $P_{0}+\epsilon P_{1}+\epsilon^{2} P_{2}+\ldots$ 


\subsection{First Order Matching}

The first order terms in $Y$ gives

$$
\begin{aligned}
u_{1}^{o, \text { inner }} & =P_{1}+\left(-\frac{w_{y}}{w} c_{1}+\frac{w_{y}}{w} Y\right) \\
u_{1}^{i, \text { outer }} & =-\frac{1}{c_{0}} Y+\frac{U(0)}{c_{0}}
\end{aligned}
$$

The terms proportional to $Y$ yield

$$
\frac{w_{y}}{w}=-\frac{1}{c_{0}}
$$

This is equivalent to, restoring the arguments to $w$ and $w_{y}$ from (26) and (27),

$$
c_{0} w_{y}\left(-c_{0}\right)+w\left(-c_{0}\right)=0
$$

In the coordinate $\eta=y-c$, we can define $\eta_{0}$ as the value of $\eta$ corresponding to $y=0$ when $c$ is approximated by $c_{0}$ :

$$
\eta_{0}=-c_{0}
$$

Recalling that $d / d \eta=d / d y$, we can rewrite the matching condition as

$$
-\eta_{0} w_{\eta}\left(\eta_{0}\right)+w\left(\eta_{0}\right)=0
$$

This is the usual Charney eigencondition.

We also have the first order match of the parts of the first order solutions which are independent of $Y$ in the overlap region.

$$
P_{1}-\frac{w_{y}}{w} c_{1}=\frac{U(0)}{c_{0}}
$$

Substituting in (64), $w_{y} / w=-1 / c_{0}$, gives

$$
P_{1}=\frac{U(0)}{c_{0}}-\frac{c_{1}}{c_{0}}
$$

Matching at second order will force $c_{1}=0$, but we will keep $c_{1}$ as an unknown for the moment.

\subsection{Second Order Matching}

Invoking $P_{0}=1$ and

$$
P_{1}=\frac{U(0)}{c_{0}}-\frac{c_{1}}{c_{0}}
$$

we obtain

$$
\begin{aligned}
u_{2}^{o, \text { inner }} & P_{2}+\left[\frac{U(0)}{c_{0}}-\frac{c_{1}}{c_{0}}\right]\left(-\frac{w_{y}}{w} c_{1}+\frac{w_{y}}{w} Y\right) \\
+ & \frac{1}{2} \frac{w_{y y}}{w} c_{1}^{2}-\frac{w_{y}}{w} c_{2}-\frac{w_{y y}}{w} c_{1} Y+\frac{1}{2} \frac{w_{y y}}{w} Y^{2}
\end{aligned}
$$

which collecting powers of $Y$ is the inner limit of the second order outer term

$$
\begin{aligned}
u_{2}^{o, \text { inner }}= & P_{2}+\left[\frac{U(0)}{c_{0}}-\frac{c_{1}}{c_{0}}\right]\left(-\frac{w_{y}}{w} c_{1}\right) \\
+ & \frac{1}{2} \frac{w_{y y}}{w} c_{1}^{2}-\frac{w_{y}}{w} c_{2}+\left\{\left[\frac{U(0)}{c_{0}}-\frac{c_{1}}{c_{0}}\right] \frac{w_{y}}{w}-\frac{w_{y y}}{w} c_{1}\right\} Y+\frac{1}{2} \frac{w_{y y}}{w} Y^{2}
\end{aligned}
$$

The outer limit of the inner solution is

$$
u_{2}^{i, \text { outerlimit }}=\left(\frac{1}{8}+\frac{r}{2 c_{0}}\right) Y^{2}+\left\{\frac{c_{1}-U(0)}{c_{0}^{2}}\right\} Y-\left\{\frac{c_{1}-U(0)}{c_{0}^{2}}\right\} U(0)
$$


Matching the terms quadratic in $Y$ yields

$$
\frac{r}{2 c_{0}}+\frac{1}{8} \sim \frac{1}{2} \frac{w_{y y}}{w}
$$

However, the confluent hypergeometric equation evaluated at $y=0$ is

$$
w_{y y}=\left(r / c_{0}+1 / 4\right) w \quad \Leftrightarrow \quad \frac{1}{2} \frac{w_{y y}}{w}=\frac{r}{2 c_{0}}+\frac{1}{8}
$$

It follows that this matching condition is automatically fulfilled.

Matching the terms in $Y$ requires, with the outer limit of the inner solution on the left,

$$
\begin{aligned}
\frac{c_{1}}{c_{0}^{2}}-\frac{U(0)}{c_{0}^{2}} & \sim \frac{c_{1}}{c_{0}^{2}}-\frac{U(0)}{c_{0}^{2}}-c_{1} \frac{w_{y y}}{w} \\
0 & \sim-c_{1} \frac{w_{y y}}{w}
\end{aligned}
$$

where the second line follows by subtracting identical terms $c_{1} / c_{0}^{2}-U(0) / c_{0}^{2}$ from both sides of the first line. Since $w_{y y}$ is zero only at isolated points, the match requires that the first order phase speed correction is zero:

$$
c_{1}=0
$$

Setting $c_{1}=0$ and showing just terms independent of $Y$ :

$$
u_{2}^{o, \text { inner } ; Y \text {-independent }}=P_{2}-\frac{w_{y}}{w} c_{2}+\frac{U(0)}{c_{0}}
$$

Substituting in (64), $w_{y} / w=-1 / c_{0}$, gives

$$
u_{2}^{o, i n n e r ; Y-i n d e n d e n d e n t}=P_{2}+\frac{c_{2}}{c_{0}}
$$

Matching the terms independent of $Y$ yields

$$
-\left\{\frac{c_{1}-U(0)}{c_{0}^{2}}\right\} U(0) \sim P_{2}+\frac{1}{c_{0}} c_{2}
$$

Applying results of two previous matches, $c_{1}=0$ and $w_{y y}=-\left(\frac{r}{-c_{0}}-\frac{1}{4}\right) w$, this simplifies to

$$
P_{2} \sim \frac{U(0)^{2}}{c_{0}^{2}}-\frac{1}{c_{0}} c_{2}
$$

Note that $c_{2}$ is as yet undetermined. Just as the first order phase speed correction was determined by a second order matching condition, similarly $c_{2}$ will fall out of the third order matching in the next subsection.

\subsection{Third Order Matching}

The matches at $O\left(y^{3}\right)$ and $O\left(y^{2}\right)$ are trivially satisfied in the sense, after replacing $c_{1}$ and $P_{2}$ by their values determined at lower orders,

$$
\begin{gathered}
O\left(y^{3}\right): \quad p_{0,3}=q_{3,3}=-1 /\left(24 c_{0}\right) \\
O\left(y^{2}\right): \quad p_{1,2}=q_{3,2}=\frac{U(0)\left(c_{0}+4 r\right)}{8 c_{0}^{2}} \\
O(y): \quad p_{2,1}=-\frac{(U(0))^{2}}{c_{0}}+\frac{c_{2}}{c_{0}^{2}}-\frac{1}{4} c_{2}-r \frac{c_{2}}{c_{0}} \\
q_{3,1}=-\frac{1}{2} \frac{v_{1}}{c_{0}}-r \frac{v_{1}}{c_{0}^{2}}+\frac{c_{2}}{c_{0}^{2}}-\frac{(U(0))^{2}}{c_{0}^{3}}
\end{gathered}
$$


Solving the equation $p_{2,1}-q_{3,1}=0$ gives the second order phase speed correction as

$$
c_{2}=v_{1}\left(1+2 \frac{r}{c_{0}}\right) \frac{2}{c_{0}+4 r}
$$

The terms that are asymptotically $y$-independent are, using an overline to denote $p_{3,0}$ and $p_{3,0}$ multiplied by a denominator-clearing common factor of $c_{0}^{3}\left(c_{0}+4 r\right)$,

$$
\begin{aligned}
& O\left(y^{0}\right): \quad \bar{p}_{3,0}=P_{3} c_{0}{ }^{4}+4 P_{3} c_{0}{ }^{3} r+2 U(0) v_{1} c_{0}+4 U(0) v_{1} r+c_{3} c_{0}^{3}+4 c_{3} c_{0}^{2} r \\
& \bar{q}_{3,0}=-2 U(0) v_{1} c_{0}-4 U(0) v_{1} r+(U(0))^{3} c_{0}+4(U(0))^{3} r-v_{0} c_{0}{ }^{3} \\
& -7 v_{0} c_{O}^{2} r-12 v_{0} c_{0} r^{2}
\end{aligned}
$$

Matching gives

$$
\begin{aligned}
c_{0}^{3}\left(c_{0}+4 r\right) P_{3}= & (U(0))^{3} c_{0}+4(U(0))^{3} r-4 U(0) v_{1} c_{0}-8 U(0) v_{1} r \\
& -v_{0} c_{0}{ }^{3}-7 v_{0} c_{0}{ }^{2} r-12 v_{0} c_{0} r^{2}-c_{3} c_{0}{ }^{3}-4 c_{3} c_{0}^{2} r
\end{aligned}
$$

The value of the third order phase speed correction and also $P_{3}$ in the amplitude multiplying the outer solution are determined by matching at fourth order. Fortunately, these do not explicitly appear in the third order inner approximation, which therefore is complete.

\section{Example One: Log(Cosh) Mean Wind}

The mean wind is

$$
\begin{aligned}
U(y ; \epsilon) & =\epsilon\{\log (\cosh (y / \epsilon))+\log (2)\} \\
& \sim|y|, \quad|y| \gg \epsilon
\end{aligned}
$$

The regularized Charney problem is

$$
u_{y y}+\left\{\frac{r-\epsilon^{-1} \operatorname{sech}^{2}(y / \epsilon)}{\epsilon\{\log (\cosh (y / \epsilon))+\log (2)\}-c}-1 / 4\right\} u=0, \quad u_{y}(0)=0, \quad u( \pm \infty)=0
$$

The outer approximation is independent-in-form of the mean wind; to all orders in $\epsilon$, the outer solution is the Whittaker function. This depends on $U(y)$ through the phase speed $c$ and the amplitude $\mathfrak{A}$ :

$$
\begin{gathered}
u^{o}(y)=\mathfrak{A} \frac{W_{r, 1 / 2}(y-c)}{W_{r, 1 / 2}\left(-c_{0}\right)} \\
c \sim c_{0}+\epsilon^{2} \frac{\pi^{2}}{12}\left(1+2 \frac{r}{c_{0}}\right) \frac{1}{c_{0}+4 r}+\ldots \\
\mathfrak{A} \sim 1+\frac{\log (2)}{c_{0}} \epsilon+P_{2} \epsilon^{2}+\ldots \\
u_{0}^{i}(Y)=1 \\
u_{1}^{i}(Y)=-\frac{1}{c_{0}} \log (\cosh (Y)) \\
u_{2}^{i}=\left(\frac{1}{8}+\frac{r}{2 c_{0}}\right) Y^{2}+A\left\{\frac{c_{1}-\log (2)}{c_{0}^{2}}\right\} \log (\cosh (Y))
\end{gathered}
$$




$$
\begin{aligned}
u_{3}^{i}= & \frac{3 \zeta(3)}{16 c_{0}}\left\{1+3 \frac{r}{c_{0}}\right\}-\frac{1}{8 c_{0}} Y^{2} \log (1+\exp (2 Y))\left\{4 \frac{r}{c_{0}}+1\right\} \\
& +\frac{\log (2)}{8 c_{0}} Y^{2}\left\{4 \frac{r}{c_{0}}+1\right\}+\frac{\pi^{2}}{96 c_{0}}\left\{4 \frac{r}{c_{0}}+1\right\} Y \\
& -\frac{1}{4 c_{0}}\left\{4 \frac{r}{c_{0}}+1\right\} Y \operatorname{Li}_{2}(-\exp (2 Y))+\frac{1}{c_{0}^{2}}\left\{c_{2}-\frac{\log ^{2}(2)}{c_{0}}\right\} \log (\cosh (Y)) \\
& +\frac{1}{32 c_{0}} \mathrm{Li}_{3}(-\exp (-2 Y))+\frac{1}{32 c_{0}}\left\{7+24 \frac{r}{c_{0}}\right\} \operatorname{Li}_{3}(-\exp (2 Y))
\end{aligned}
$$

where $\mathrm{Li}_{n}$ is a polylogarithm funcion, defined by (112) in the first appendix.

Fig. 3 shows the different orders in the inner solution for the log-of-cosh regularization. Only a small range in the inner coordinate $Y$ is shown because the regularized wind $\log (\cosh (Y))$ decays to a linear profile as $O(\exp (-2 Y))$, and is thus within a quarter of a percent of its asymptote for $Y=3$. Although the cubic growth of $u_{3}^{i}$ is not obvious on the limited range shown,

$$
u_{n}^{i} \sim \text { constant }|Y|^{n}, \quad|Y| \rightarrow \infty
$$

The power-law growth of the inner order terms is not a pathology, but rather is essential so that the inner solution matches the inner limit of the outer solution, which is a power series in $Y$, or at leading order a power series in $\epsilon Y$.

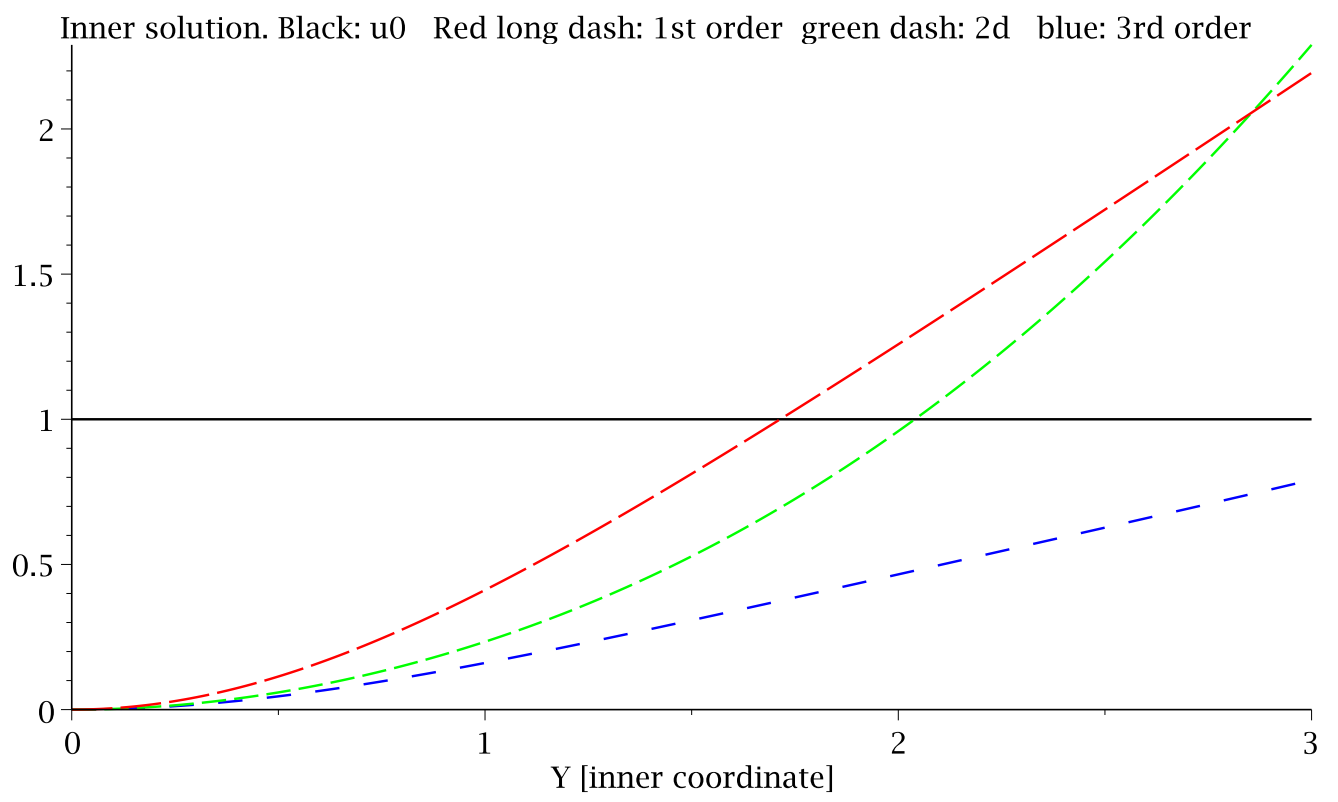

Figure 3: The lowest four orders of the inner solution (after matching) for $r=1 / 2$ and $U(y)=\epsilon \log (2 \cosh (y / \epsilon))$. Solid: $u_{0}^{i} \equiv 1$. Long red dash: first order. Short green dash: $u_{2}^{i}$. Medium-length blue dash: third order. 


\section{Example Two: The Linear-Erf Jet}

The "linear-erf" jet is defined by

$$
\begin{gathered}
U(y ; \epsilon)=\epsilon\{(y / \epsilon)) \operatorname{erf}(y / \epsilon)\} \\
\sim|y|, \quad|y| \gg \epsilon \\
u_{y y}+\left\{\frac{r-\epsilon^{-1}[4 / \sqrt{\pi}]\left(1-(y / \epsilon)^{2}\right) \exp \left(-(y / \epsilon)^{2}\right)}{y \operatorname{erf}(y / \epsilon)-c}-1 / 4\right\}=0, u_{y}(0)=0, u( \pm \infty)=0 \\
u_{0}^{i}(Y)=1 \\
u_{1}^{i}(Y)=-\frac{1}{c_{0}} Y \operatorname{erf}(Y) \\
u_{2}^{i}=\left(\frac{1}{8}+\frac{r}{2 c_{0}}\right) Y^{2} \\
-\frac{1}{c_{0}}\left\{\frac{1}{8}+\frac{r}{2 c_{0}}\right\}\left\{Y^{2} U(Y)-4 Y V_{Y}+6 V(Y)\right\} \\
c=c_{0}^{i}+\epsilon^{2} c_{2}+\ldots
\end{gathered}
$$

where

$$
\begin{gathered}
c_{2}=-\frac{1}{2}\left(1+2 \frac{r}{c_{0}}\right) \frac{1}{c_{0}+4 r} \\
V(Y)=(1 / 12)\left(2 Y^{3}-3 Y\right) \operatorname{erf}(Y)+(1 /[6 \sqrt{\pi}]) Y^{2} \exp \left(-Y^{2}\right)
\end{gathered}
$$

In the outer limit,

$$
V(Y) \sim(1 / 6) Y^{3}-(1 / 4) Y \quad Y \gg 1
$$

For $\epsilon=1$, the corrections are as large as the unperturbed solution, so numerical solutions are used to extend regularized/unregularized comparisons to $\epsilon=1$ in the next section.

\section{Numerical Comparison of the Charney Solution with the Regularized Solution with $\epsilon=1$}

The numerical calculations were computed using a rational Chebyshev pseudospectral method as described in $[3,6]$. The solution is computed in the form of a Fourier series but with rational Chebyshev functions replacing the sines and the cosines:

$$
u(y)=\sum_{n=0}^{\infty} a_{n} \operatorname{TL}_{n}(y)
$$

where the explicit form of the TL functions is given in [3]. Fig. 4 shows the computed coefficients $a_{n}$. The plot is consistent with the theoretical prediction that the coefficients are proportional to $\exp \left(-q n^{1 / 2}\right)$ for some constant $q$ [3-5]; the error when the series is truncated after the $N$-th term is therefore proportional to $\exp (-q \sqrt{N})$ as explained in Chapter 2 of [4]. 


\section{TL coefficients $\mathrm{L}=1$ Charney: black epsilon=1: red}

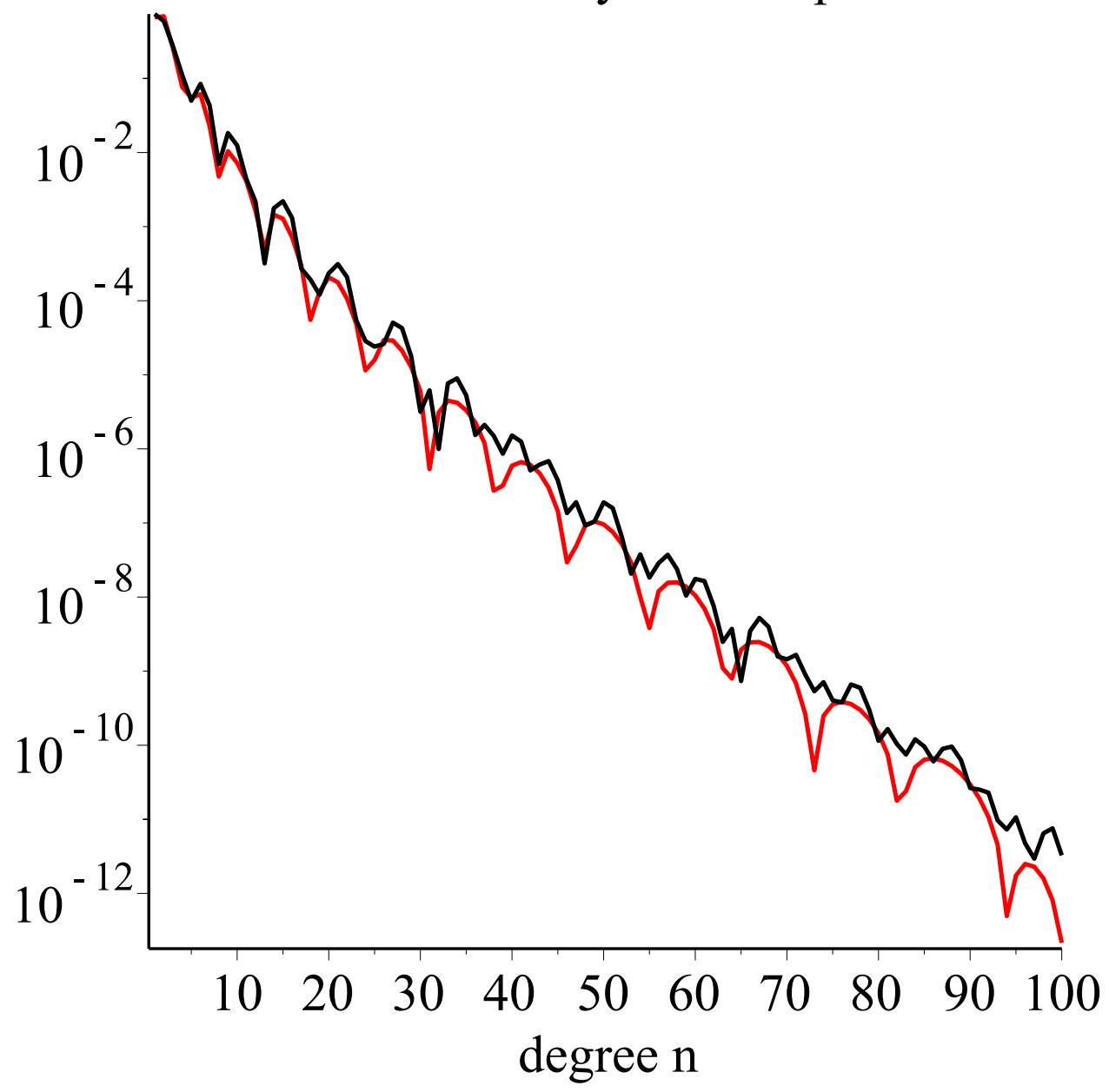

Figure 4: Comparison, for $r=1 / 2$ and map parameter $L=1$, of the TL coefficients for the Charney eigenfunction [black] with the corresponding coefficients of the $\log (\cosh )$ regularization of the Charney problem for $\epsilon=1$.

Fig. 5 shows that the reflection of Charney's mode about $y=0$ has a discontinuous slope because $\psi_{y}(0) \neq$ 0 . In contrast, the slope of the regularized solutions is always zero at the origin and the reflection is smooth and singularity-free.

For $r=1 / 2$, the complex phase speeds are (from rational Chebyshev numerical solutions)

$$
\begin{aligned}
c_{\text {Charney }} & =0.901016078298+0.544989507567 i \\
c(\epsilon=1 / 4) & =0.928711457199+0.530656566091 i \\
c(\epsilon=1 / 2) & =0.9945303130+0.4819021245 i \\
c(\epsilon=1) & =1.1430477218+0.3217382311 i
\end{aligned}
$$




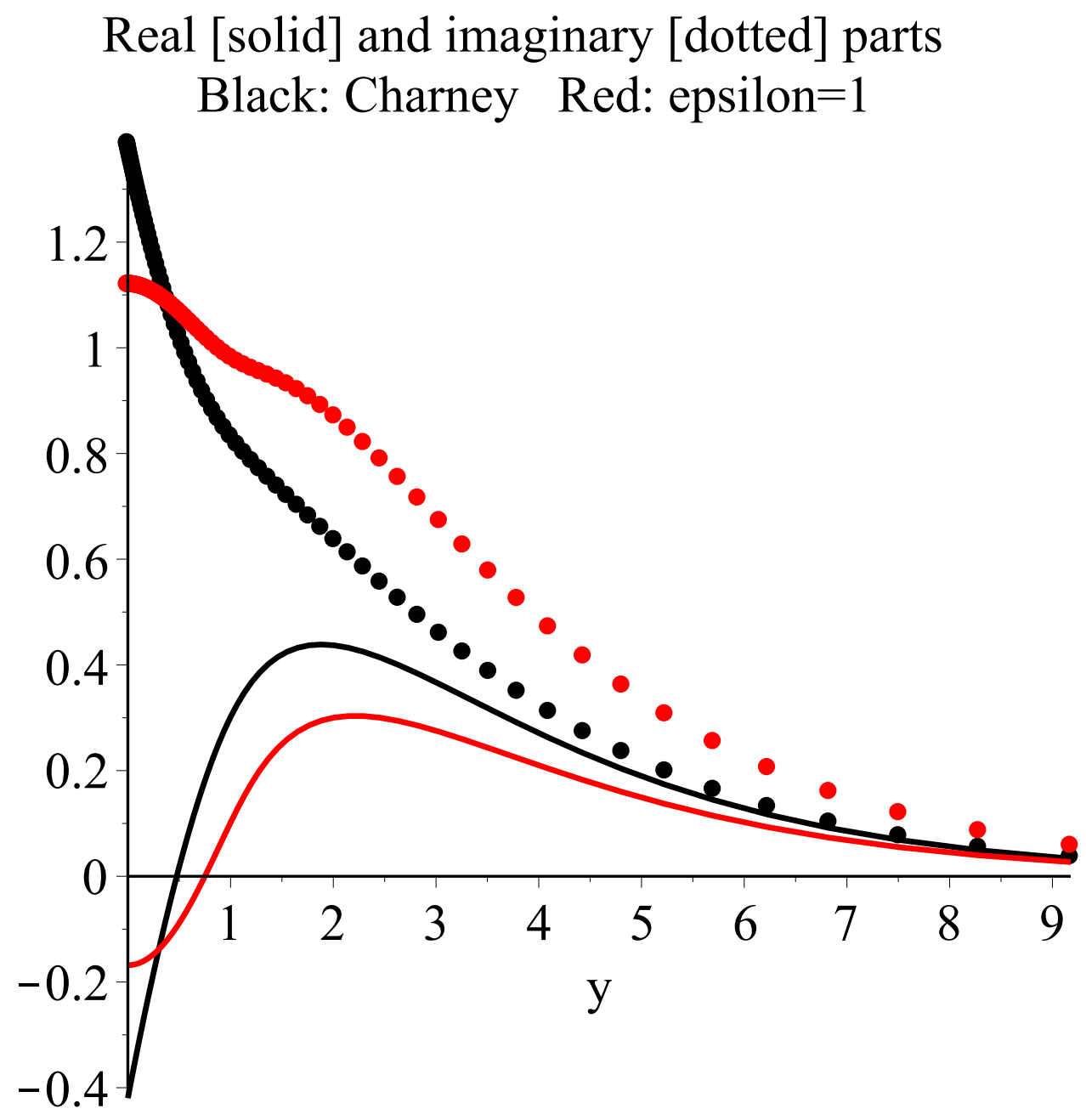

Figure 5: Comparison of Charney eigenfunction with $\log (\cosh )$ regularization for $\epsilon=1$.

The absolute value of the complex modes shows little change (Fig. 6). When normalized properly, meaning that the coefficients of $\mathrm{TL}_{0}$ are the same for both the Charney and regularized modes, the real and imaginary parts are also similar.

\section{Over-reflection Theory}

Fig 7 is a schematic of the Lindzen-Tung-Farrell-Rosenthal Wave Overreflection Theory (OR). The bottom of the diagram is the ground (for baroclinic instability) or the center of the symmetric "point jet" (for barotropic instability).

In OR theory, waves propagate from the ground upward to a turning point. This turning point is ESSENTIAL because it stops the wave propagation and allows the waves to tunnel to the critical level. Lindzen et al. assert that it is not possible for waves to propagate directly to the critical level (or latitude), there to be overreflected, because the vertical group velocity goes to zero at the critical level, so the waves would never make it as oscillations, but only by tunneling through an interval of exponential growth away from the critical level. ("Tunneling" is used in the same meaning as in quantum mechanics.) The turning point above the critical level, which creates the region of exponential decay upwards to infinite height, is OPTIONAL. This 


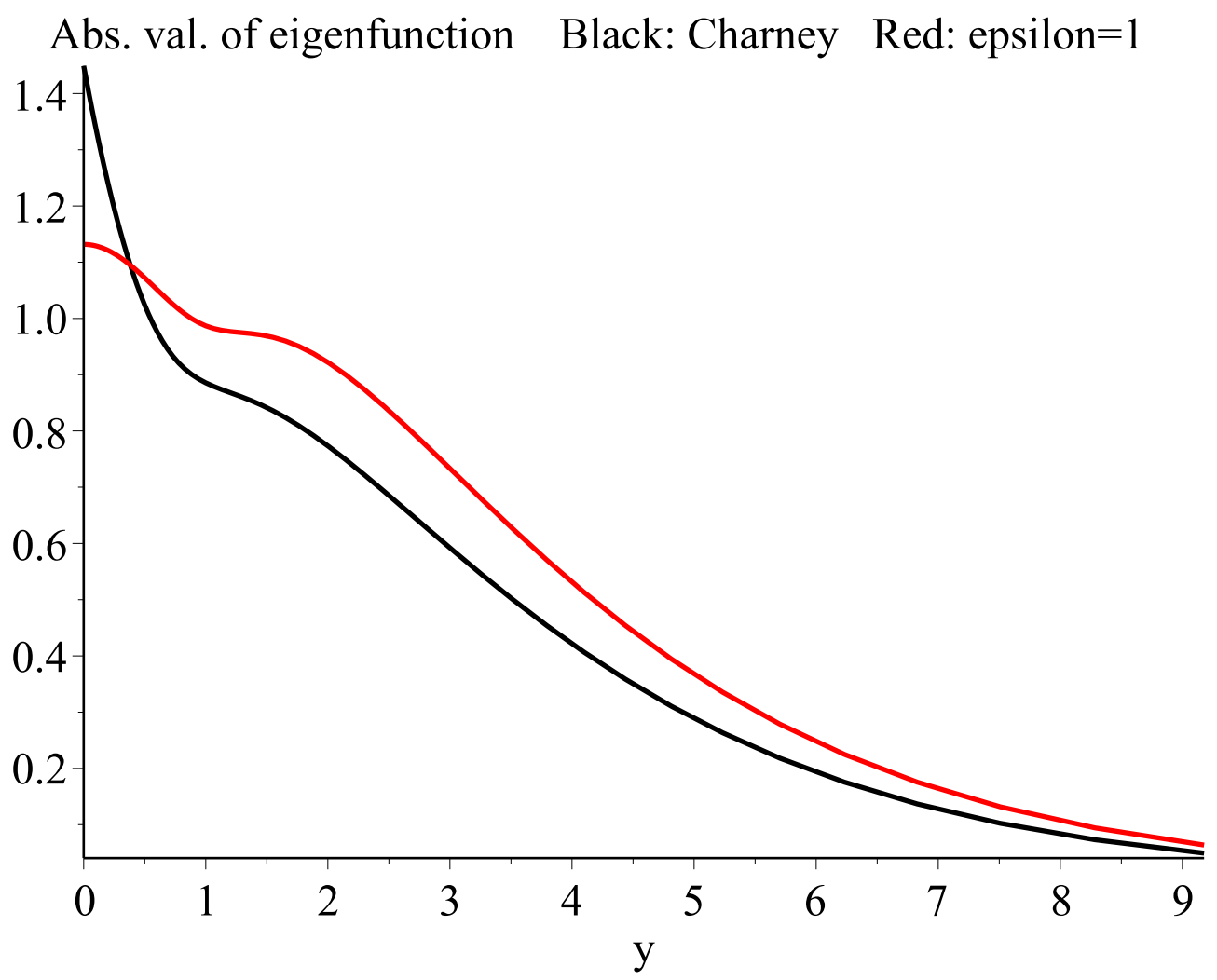

Figure 6: Comparison of the absolute value of the Charney eigenfunction with $\log (\cosh )$ regularization for $\epsilon=1$.

turning point could be replaced by a rigid upper boundary without modifying the overreflection process, and is therefore labeled "irrelevant". The idea is that waves from the ground are overreflected downward from the critical level, bounce off the ground, and are overreflected again so as to build up an amplifying normal mode in a process reminiscent of a laser signal growing through repeated bounces within a ruby crystal.

Lindzen's review [19] shows that the theory also applies to gravity waves governed by the Taylor-Goldstein equation and its invariants, but this is outside the scope of the present discussion.

The first obvious difficulty with OR theory is that the region of waves near the ground, the "resonance cavity" of the laser analogy, is compressed into a delta function. The inner solution of the matched asymptotic expansion approximates the solution in this wavy region, number "1" in the schematic, by the inner approximation. However, the leading order inner solution is just the constant, which is as far from a wave as one could possibly imagine. The first-order correction is merely the mean wind $U(y)$. This does curve, but speedily and monotonically asymptotes to a linear function. The first order inner approximation is not very wavy either.

\section{Implications}

The delta functions in the Charney problem and barotropic point-jet, so worrisome to J. S. A. Green in the quote at the beginning of the article, have been removed. The regularized solutions confirm the soundness of the point-jet model of Lindzen, Rosenthal and Farrell [23]. In particular the lowest order eigenvalue is identical with theirs. The eigenfunctions are the same, too, to all orders everywhere outside the inner region of width $O(\epsilon)$ around the jet maximum. 


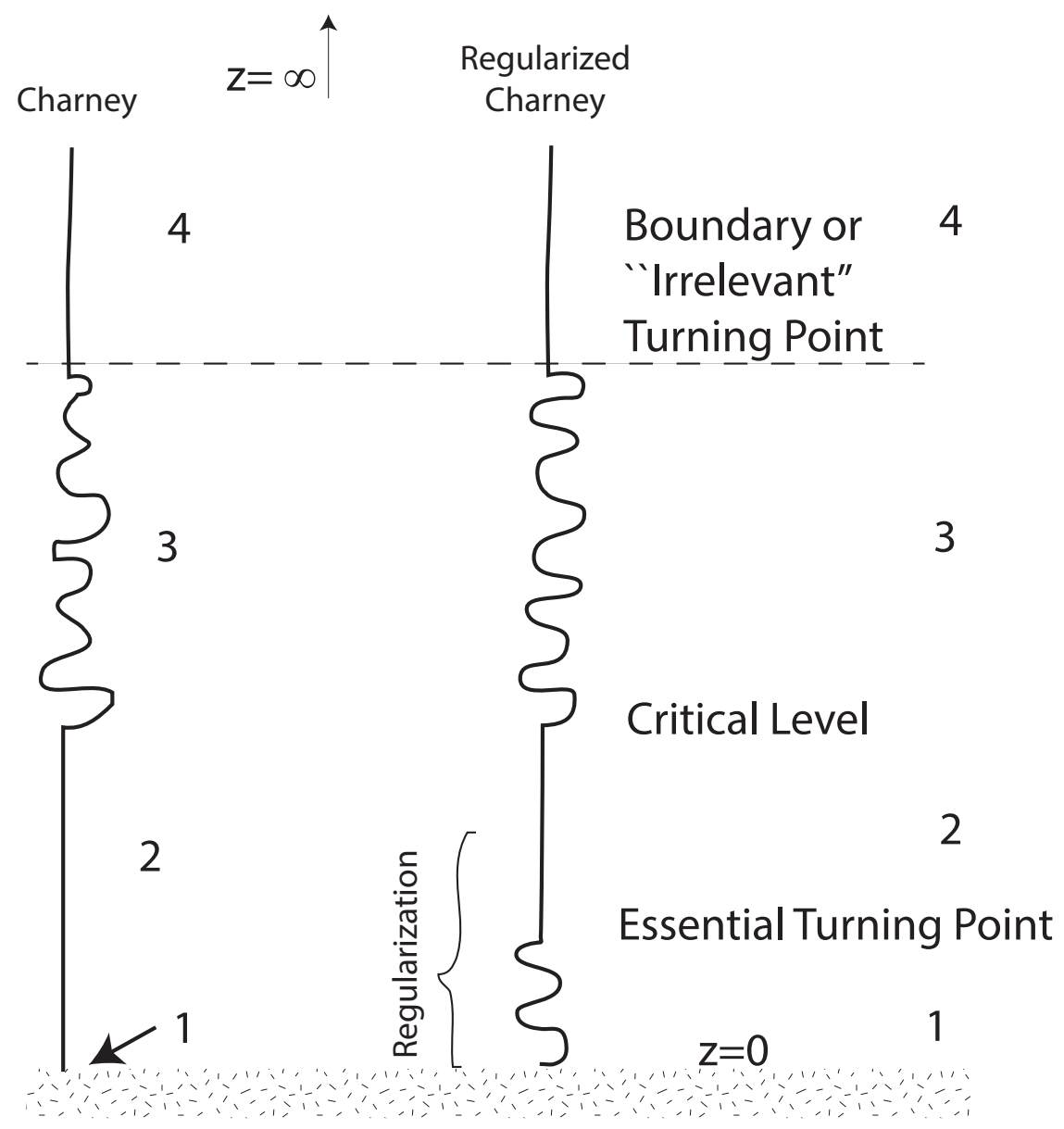

Figure 7: Schematic of Overreflection Theory, similar to Fig. 1 of Lindzen and Tung [24]. The wiggly lines denote regions of wave propagation; straight vertical lines denote regions of exponential growth or decay in space. Far from the ground or the center of the point jet, the wave is confined by either a turning point with exponential decay on the far side, as true of the Charney problem, or a wall boundary, as marked by the horizontal dashed line (top of the figure). The numbers on the far right refer to the corresponding numbered regions in Lindzen and Tung's article. In the Charney problem, the all-important oscillation region " 1 " - the "resonant cavity" of the "laser" - is squished down into a layer of INFINITESIMAL THICKNESS where the curvature of the mean wind is a DELTA FUNCTION.

Yet larger issues remain. A linear shear that is linear everywhere, and not merely piecewise-linear, is always stable. Therefore the inner region of width $\epsilon$, which may be arbitrarily thin, somehow controls the dynamics everywhere. And yet the Overreflection Theory completely ignores the physics in the inner region, and analyzes instability entirely in terms of properties of the outer region. Even so, the outer region is completely impotent to create instability on its own. The inner region is both all-powerful and irrelevant!

We shall return to these mysteries after analyzing the WKB-thinking that underlies OR theory in the third part of this series.

Acknowledgement: This work was supported by the NSF through grants OCE 0451951, OCE 1059703 and DMS1521158. I thank the three reviewers. 


\section{A Dilogs, Polylogarithms and the log(cosh) Function}

\section{A.1 Definitions}

The matched asymptotic expansions require the unfamiliar transcendentals known as the "polylogarithms" defined by

$$
\operatorname{Li}_{m}(z) \equiv \sum_{n=1}^{\infty} \frac{z^{n}}{n^{m}}
$$

$\mathrm{Li}_{2}(x)$ is the "dilogarithm", often written simply as Li. Table 1 shows that the integrals and derivatives of the $\log (\cosh )$ function can be expressed in terms of the polylogarithms and ordinary hyperbolic functions. This allows explicit, analytical expressions for the lowest three orders of the $\epsilon$ series for the regularized Charney problem.

The next two subsections solve subproblems essential to the third order solution. An important simplification is that the inner approximation is symmetric about the origin to all orders.

An arbitrary function is said to be "symmetric with respect to the origin" if and only if it has the property that $f(-Y)=f(Y)$ for all $Y$. The integrands for both $\diamond$ and $\mho$, the functions derived in the rest of this section, have this symmetry. The following helpful lemma is a slight variation on the analysis of Chapter 9 of [4]. It may also be proved by observing that all odd powers of $Y$ are antisymmetric with respect to the origin, all even powers are symmetric, and then expanding the integrands in power series.

Lemma 1. The integrand of a symmetric function is antisymmetric except for the arbitrary constant of integration. The integral-of-the-integral of a symmetric function is symmetric except for the additive term $C Y$ where $C$ is a constant of integration.

Table 1: Derivatives and Integrals of the $\log (\cosh )$ Function

The $p_{j}$ are constants of integration.

\begin{tabular}{cc}
\hline$U_{Y Y}$ & $\begin{array}{c}\operatorname{sech}^{2}(Y) \\
\tanh (Y)\end{array}$ \\
\hline$U$ & $\log (\cosh (Y))+\log (2)$ \\
\hline $\int U\left(Y^{\prime}\right) d Y^{\prime}$ & $p_{0}+(1 / 2) Y^{2}+(1 / 2) \operatorname{Li}_{2}(-\exp (-2 Y))$ \\
\hline $\int d Y^{\prime \prime} \int^{Y^{\prime \prime}} U\left(Y^{\prime}\right) d Y^{\prime}$ & $p_{0}+p_{1} Y+(1 / 6) Y^{3}-(1 / 4) \mathrm{Li}_{3}(-\exp (-2 Y))$ \\
\hline$m$-th iterated integral of $U$ & $P_{m-1}(Y)+(1 /(m+1) !) Y^{m+1}-(-1 / 2)^{m} \operatorname{Li}_{m+1}(-\exp (-2 Y))$ \\
& $P_{m-1}$ with coefficients $p_{j}$ is an arbitrary polynomial \\
& of degree $(m-1) . \operatorname{Li}_{m}(z) \equiv \sum_{n=1}^{\infty} \frac{z^{n}}{n^{m}}$. \\
\hline
\end{tabular}




\section{A.2 Analytical evaluation of $\diamond(Y)$, the second integral of $\log (\cosh (Y))$ [ part of the third order solution]}

Theorem 1. 1 .

$$
\begin{aligned}
\diamond\left(Y ; p_{0}, p_{1}\right) & \equiv \int^{Y} d Y^{\prime \prime} \int^{\prime \prime} \log \left(\cosh \left(Y^{\prime}\right)\right) d Y^{\prime} \\
& =p_{0}+p_{1} Y-\frac{1}{2} \log (2) Y^{2}+\frac{1}{6} Y^{3}-\frac{1}{4} \operatorname{Li}_{3}(-\exp (-2 Y))
\end{aligned}
$$

where $p_{0}$ and $p_{1}$ are arbitrary integration constants.

2. The solution to

$$
\diamond_{Y Y}(Y)=\log (\cosh (Y)), \quad \diamond(0)=\diamond_{Y}(0)=0
$$

is symmetric with respect to $Y=0$ and may be expressed in either of the two equivalent forms:

$$
\begin{aligned}
\diamond\left(Y ;-\frac{3}{16} \zeta(3), \frac{\pi^{2}}{24}\right) & =-\frac{3}{16} \zeta(3)+\frac{\pi^{2}}{24} Y-\frac{1}{2} \log (2) Y^{2}+\frac{1}{6} Y^{3}-\frac{1}{4} \operatorname{Li}_{3}(-\exp (-2 Y)) \\
& =-\frac{3}{16} \zeta(3)-\frac{1}{2} \log (2) Y^{2}-\frac{1}{8} \mathrm{Li}_{3}(-\exp (-2 Y))-\frac{1}{8} \operatorname{Li}_{3}(-\exp (2 Y))
\end{aligned}
$$

Proof:

$$
\begin{aligned}
\log (\cosh (Y)) & =-\log (2)+Y+\log (1+\exp (-2 Y)) \\
& =-\log (2)+Y+\sum_{n=1}^{\infty}(-1)^{n+1} \frac{\exp (-2 n Y)}{n}, \quad Y>0
\end{aligned}
$$

Term-by-term indefinite integration gives

$$
\begin{aligned}
\int \log \left(\cosh \left(Y^{\prime}\right)\right) d Y^{\prime} & =p_{1}-\log (2) Y+\frac{1}{2} Y^{2}-\frac{1}{2} \sum_{n=1}^{\infty}(-1)^{n+1} \frac{\exp (-2 n Y)}{n^{2}}, \quad Y>0 \\
& =p_{1}-\log (2) Y+\frac{1}{2} Y^{2}+\frac{1}{2} \sum_{n=1}^{\infty} \frac{[-\exp (-2 Y)]^{n}}{n^{2}}, \quad Y>0 \\
& =p_{1}-\log (2) Y+\frac{1}{2} Y^{2}+\frac{1}{2} \operatorname{Li}_{2}(-\exp (-2 Y))
\end{aligned}
$$

where $p_{1}$ is the arbitrary integration constant.

Integrating again gives

$$
\begin{aligned}
\int d Y^{\prime} \int \log \left(\cosh \left(Y^{\prime}\right)\right) d Y^{\prime} & =\int^{Y} d Y^{\prime}\left\{p_{1}-\log (2) Y^{\prime}+\frac{1}{2} Y^{\prime 2}+\frac{1}{2} \sum_{n=1}^{\infty} \frac{\left[-\exp \left(-2 Y^{\prime}\right)\right]^{n}}{n^{2}}\right\}, \quad Y>0 \\
& =p_{0}+p_{1} Y-\frac{1}{2} \log (2) Y^{2}+\frac{1}{6} Y^{3}-\frac{1}{4} \sum_{n=1}^{\infty} \frac{[-\exp (-2 Y)]^{n}}{n^{3}}, \quad Y>0 \\
& =p_{0}+p_{1} Y-\frac{1}{2} \log (2) Y^{2}+\frac{1}{6} Y^{3}-\frac{1}{4} \operatorname{Li}_{3}(-\exp (-2 Y))
\end{aligned}
$$

where $p_{0}$ and $p_{1}$ are arbitrary integration constants.

The solution to

$$
\diamond_{Y Y}(Y)=\log (\cosh (Y)), \quad \diamond(0)=\diamond_{Y}(0)=0
$$

requires the power series expansion

$$
\diamond\left(Y ; p_{0}, p_{1}\right)=p_{0}+\frac{3}{16} \zeta(3)+\left(p_{1}-\frac{\pi^{2}}{24}\right) Y-\frac{1}{2} \log (2) Y^{2}+\ldots
$$


where we have used $\zeta(m)=\sum_{n=1}^{\infty} 1 / n^{m}$ and $\zeta(2)=\pi^{2} / 6$. The solution is

$$
\begin{aligned}
\diamond\left(Y ;-\frac{3}{16} \zeta(3), \frac{\pi^{2}}{24}\right) & =-\frac{3}{16} \zeta(3)+\frac{\pi^{2}}{24} Y-\frac{1}{2} \log (2) Y^{2}+\frac{1}{6} Y^{3}+\ldots \\
& =-\frac{3}{16} \zeta(3)-\frac{1}{2} \log (2) Y^{2}-\frac{1}{8} \operatorname{Li}_{3}(-\exp (-2 Y))-\frac{1}{8} \operatorname{Li}_{3}(-\exp (2 Y))
\end{aligned}
$$

where the second line follows from the first after (i) recognizing that the first form is symmetric with respect to the origin by invoking Lemma 1 and noting that the boundary condition $d \diamond / d Y(0)=0$ has removed the term linear in $Y$ and (ii) applying the formula for the symmetric part of an arbitrary function, $\operatorname{Symm}(f(x))=$ $(1 / 2)[f(x)+f(-x)]$. Both forms are identical; the first does not look symmetric in $Y$, though it is, but this apparently unsymmetric form is convenient for asymptotic matching. The second is self-evidently symmetric about $Y=0$, but hides the fact that $\diamond(Y)$ has linear and cubic terms as $Y \rightarrow \infty$.

\section{A.3 Analytical evaluation of $\mho(Y)$, the iterated integral of $Y^{2} \operatorname{sech}^{2}(Y)$, [another part of the third order solution]}

$$
\int d Y^{\prime} \int Y^{\prime 2} \operatorname{sech}^{2}\left(Y^{\prime}\right) d Y^{\prime}=\mho\left(Y ; q_{0}, q_{1}\right)
$$

where $q_{0}, q_{1}$ ) are constants of integration.

$$
\begin{aligned}
\int Y^{\prime 2} \operatorname{sech}^{2}\left(Y^{\prime}\right) d Y^{\prime}= & q_{1}-\frac{1}{12} \frac{\pi^{2}}{1+\left(\mathrm{e}^{Y}\right)^{2}}-1 / 12 \frac{\pi^{2}\left(\mathrm{e}^{Y}\right)^{2}}{1+\left(\mathrm{e}^{Y}\right)^{2}}+2 \frac{Y^{2}\left(\mathrm{e}^{Y}\right)^{2}}{1+\left(\mathrm{e}^{Y}\right)^{2}} \\
& -2 \frac{Y \ln \left(1+\mathrm{e}^{2 Y}\right)}{1+\left(\mathrm{e}^{Y}\right)^{2}}-2 \frac{Y \ln \left(1+\mathrm{e}^{2 Y}\right)\left(\mathrm{e}^{Y}\right)^{2}}{1+\left(\mathrm{e}^{Y}\right)^{2}} \\
& -\frac{\mathrm{Li}_{2}\left(2,-\left(\mathrm{e}^{Y}\right)^{2}\right)}{1+\left(\mathrm{e}^{Y}\right)^{2}}-\frac{\mathrm{Li}_{2}\left(2,-\left(\mathrm{e}^{Y}\right)^{2}\right)\left(\mathrm{e}^{Y}\right)^{2}}{1+\left(\mathrm{e}^{Y}\right)^{2}} \\
= & \tilde{q}_{1}-\frac{\pi^{2}}{12}+2 Y^{2} \frac{\exp (2 Y)}{1+\exp (2 Y)}-2 Y \log (1+\exp (2 Y))-\operatorname{Li}_{2}(-\exp (2 Y))
\end{aligned}
$$

where $\tilde{q}_{1}$ is a constant of integration.

Term-by-term integration and $q_{1} \equiv \tilde{q}_{1}-\left(\pi^{2} / 12\right)$ yield

$$
\begin{aligned}
\mho\left(Y ; q_{0}, q_{1}\right) & =\int^{Y}\left\{q_{1}+2 Y^{2} \frac{\exp (2 Y)}{1+\exp (2 Y)}-2 Y \log (1+\exp (2 Y))-\operatorname{Li}_{2}(-\exp (2 Y))\right\} d Y^{\prime} \\
& \left.\left.=q_{0}+q_{1} Y+Y^{2} \ln \left(1+\mathrm{e}^{2 Y}\right)+2 Y \operatorname{Li}_{2}(-\exp (2 Y))\right)-\frac{3}{2} \operatorname{Li}_{3}(-\exp (2 Y))\right)
\end{aligned}
$$

where we have used

$$
\begin{gathered}
\int 2 Y^{\prime 2} \frac{\exp \left(2 Y^{\prime}\right)}{1+\exp \left(2 Y^{\prime}\right)} d Y^{\prime}=Y^{2} \log (1+\exp (2 Y))+Y \operatorname{Li}_{2}(-\exp (2 Y))-(1 / 2) \operatorname{Li}_{3}(-\exp (2 Y)) \\
\quad \int^{Y}(-2) Y^{\prime} \log \left(1+\exp \left(2 Y^{\prime}\right)\right) d Y^{\prime}=Y \operatorname{Li}_{2}(-\exp (2 Y))-(1 / 2) \operatorname{Li}_{3}(-\exp (2 Y))
\end{gathered}
$$




$$
\int^{Y}(-1) \operatorname{Li}_{2}\left(-\exp \left(2 Y^{\prime}\right)\right) d Y^{\prime}=(-1 / 2) \operatorname{Li}_{3}(-\exp (2 Y))
$$

We need the specific function that solves the ODE and also two homogenous boundary conditions:

$$
\begin{gathered}
\mho_{Y Y}(Y)=Y^{2} \operatorname{sech}^{2}(Y), \quad \mho(0)=\mho_{Y}(0)=0 \\
\mho\left(0 ; q_{0}, q_{1}\right)=q_{0}-\frac{3}{2} \operatorname{Li}_{3}(-1)
\end{gathered}
$$

$\operatorname{Li}_{3}(-1)=\sum_{n=1}^{\infty}(-1)^{n} \frac{1}{n^{3}}=-(3 / 4) \zeta(3)$,

$$
\begin{gathered}
q_{0}=\frac{3}{2} \mathrm{Li}_{3}(-1)=-\frac{9}{8} \zeta(3)=-1.3523140160545435710747 \\
\frac{d \mho}{d Y}\left(0 ; q_{0}, q_{1}\right)=q_{1}-\mathrm{Li}_{2}(-1)
\end{gathered}
$$

$\operatorname{Li}_{2}(-1)=\sum_{n=1}^{\infty}(-1)^{n} / n^{2}=-\pi^{2} / 12$. Therefore

$$
\begin{gathered}
q_{1}=\operatorname{Li}_{2}(-1)=-\frac{\pi^{2}}{12}=-.822467033424113218236207583 \\
\mho\left(0 ;-\frac{9}{8},-\frac{\pi^{2}}{12}\right)=(3 / 4) \zeta(3)+\frac{\pi^{2}}{12} Y+Y^{2} \ln \left(1+\mathrm{e}^{2 Y}\right)+2 Y \operatorname{Li}_{2}(-\exp (2 Y))-\frac{3}{2} \operatorname{Li}_{3}(-\exp (2 Y))
\end{gathered}
$$

We need the following formulas, easily obtained by asymptotic and power series expansions in Maple, to derive the outer limit of the inner solution.

$$
\begin{aligned}
\log (\cosh (Y) & \sim Y-\log (2)+\exp (-2 Y)-\frac{1}{2} \exp (-4 Y)+O(\exp (-6 Y)) \\
\mathrm{Li}_{2}(-\exp (-2 Y)) & \sim-\mathrm{e}^{-2 Y}+1 / 4 \mathrm{e}^{-4 Y}-1 / 9 \mathrm{e}^{-6 Y}+\ldots \\
\mathrm{Li}_{2}(-\exp (2 Y)) & \sim-2 Y^{2}-1 / 6 \pi^{2}+\mathrm{e}^{-2 Y}-1 / 4 \mathrm{e}^{-4 Y}+1 / 9 \mathrm{e}^{-6 Y} \\
\mathrm{Li}_{3}(-\exp (-2 Y)) & \sim-\mathrm{e}^{-2 Y}+\frac{1}{8} \mathrm{e}^{-4 Y}-\frac{1}{27} \mathrm{e}^{-6 Y} \\
\mathrm{Li}_{3}(-\exp (2 Y)) & \sim-\frac{\pi^{2}}{3} Y-\frac{4}{3} Y^{3}-\mathrm{e}^{-2 Y}+\frac{1}{8} \mathrm{e}^{-4 Y}-\frac{1}{27} \mathrm{e}^{-6 Y}+\ldots \\
\log (1+\exp (2 Y)) & \sim 2 Y+\exp (-2 Y)-\frac{1}{2} \exp (-4 Y)+\frac{1}{3} \exp (-6 Y)
\end{aligned}
$$

\section{B Linear-Error Function Identities and Lemma}

Table 2 gives the identities which are needed when the mean flow is regulzarized using the error function.

The solution to

$$
V_{Y Y}=U, \quad V(0)=V_{Y}(0)=0
$$

is

$$
V(Y)=(1 / 12)\left(2 Y^{3}-3 Y\right) \operatorname{erf}(Y)+(1 /[6 \sqrt{\pi}]) Y^{2} \exp \left(-Y^{2}\right)
$$

\section{References}

[1] N. A. Bakas and B. F. Farrell, The role of nonnormality in overreflection theory, J. Atmos. Sci., 67 (2010), pp. $2457-276$. 
Table 2: Derivatives and Integrals of the $Y \operatorname{erf}(Y)$ Function

\begin{tabular}{cc}
\hline$U_{Y Y}$ & $(4 / \sqrt{\pi})\left(1-Y^{2}\right) \exp \left(-Y^{2}\right)$ \\
\hline$U_{Y}$ & $\operatorname{erf}(Y)+(2 / \sqrt{\pi}) Y \exp \left(-Y^{2}\right)$ \\
\hline$U$ & $A^{\prime}+(1 / 2)\left(Y^{2}-1 / 2\right) \operatorname{erf}(Y)+(1 /[2 \sqrt{\pi}]) Y \exp \left(-Y^{2}\right)$ \\
\hline $\int U\left(Y^{\prime}\right) d Y^{\prime}$ & $A^{\prime \prime}+B^{\prime \prime} Y+(1 / 12)\left(2 Y^{3}-3 Y\right) \operatorname{erf}(Y)+(1 /[6 \sqrt{\pi}]) Y^{2} \exp \left(-Y^{2}\right)$ \\
& where $A^{\prime}, A^{\prime \prime}, B^{\prime \prime}$ are arbitrary constants. \\
\hline
\end{tabular}

[2] J. P. Boyd, The continuous spectrum of linear Couette flow with the beta effect, Journal of the Atmospheric Sciences, 40 (1983), pp. 2304-2308.

[3] - Orthogonal rational functions on a semi- infinite interval, J. Comput. Phys., 70 (1987), pp. 63-88.

[4] - Chebyshev and Fourier Spectral Methods, Dover, New York, 2001. 680 pp.

[5] - Large-degree asymptotics and exponential asymptotics for Fourier coefficients and transforms, Chebyshev and other spectral coefficients, J. Engrg. Math., 63 (2009), pp. 355-399.

[6] _ Five Themes in Chebyshev Spectral Methods Applied to the Regularized Charney Eigenproblem: Extra Numerical Boundary Conditions, a Boundary-Layer-Resolving Change of Coordinate, Parameterizing a Curve Which Is Singular at an Endpoint, Extending the Tau Method to Log-and-Polynomials anf Finding the Roots of a Polynomial-and-Log Approximation, Comput. Math. Applic., 71 (2016), pp. 1227-1241.

[7] F. P. Bretherton, Baroclinic instability and the short wavelength cut-off in terms of potential vorticity, Quart. J. Roy. Meteor. Soc., 92 (1966), pp. 335-345.

[8] _ Critical layer instability in baroclinic flows, Quart. J. Roy. Meteor. Soc., 92 (1966), pp. 325-334.

[9] J. G. Charney, The dynamics of long waves in a baroclinic westerly current, J. Meteor., 4 (1947), pp. 135-162.

[10] B. F. Farrell, Transient development in confluent and diffluent flow, J. Atmos. Sci., 46 (1989), pp. 3279-3288.

[11] J. S. A. Green, A problem in baroclinic stability, Quart. J. Roy. Meteor. Soc., 86 (1960), pp. 237-251.

[12] J. S. A. Green, Critical layer instability in baroclinic flows" and "baroclinic instability and the short wavelength cutoff in terms of potential vorticity, Quarterly Journal of the Royal Meteorological Society, 103 (1967), pp. 552-552.

[13] N. Harnik and E. Heifetz, Relating overreflection and wave geometry to the counterpropagating Rossby wave perspective: Toward a deeper mechanistic understanding of shear instability, J. Atmos. Sci., 64 (2007), pp. 2238-2261.

[14] E. Heifetz, C. H. Bishop, and P. Alpert, Counter-propagating Rossby waves in the barotropic Rayleigh model of shear instability, Quart. J. Royal Meteor. Soc., 125, Part B (1999), pp. 2835-2853.

[15] E. Heifetz, C. H. Bishop, B. J. Hoskins, and J. Methven, The counter-propagating rossby-wave perspective on baroclinic instability. I: Mathematical basis, Quart. J. Royal Meteor. Soc., 130, Part A (2004), pp. 211-231.

[16] E. Heifetz, N. Harnik, and T. Tamarin, Canonical Hamiltonian representation of pseudoenergy in shear flows using counterpropagating Rossby waves, Quart. J. Royal Meteor. Soc., 135 (2009), pp. 2161-2167.

[17] E. Heifetz, Y. Reuveni, A. Gelfgat, E. Kit, and J. Methven, The counterpropagating Rossby wave perspective on Kelvin Helmholtz instability as a limiting case of a Rayleigh shear layer with zero width, Phys. Fluids, 18 (2006), p. 018101.

[18] H.-L. Kuo, Three-dimensional disturbances in a baroclinic zonal current, J. Meteor., 9 (1952), pp. 260-278.

[19] R. S. Lindzen, Instability of plane parallel shear flow (toward a mechanistic picture of how it works), Pure and Applied Geophysics, 126 (1988), pp. 103-121.

[20] Dynamics in Atmospheric Physics, Cambridge University Press, New York, 1990. 310 pp.

[21] R. S. Lindzen, B. Farrell, and K. K. Tung, The concept of wave overreflection and its application to baroclinic instability, J. Atmos. Sci., 37 (1980), pp. 44-63.

[22] R. S. Lindzen and A. J. Rosenthal, $\underline{A}$ WKB asymptotic analysis of baroclinic instability, J. Atmos. Sci., 38 (1981), pp. 619-629.

[23] R. S. Lindzen, A. J. Rosenthal, and B. Farrell, Charney's problem for baroclinic instability applied to barotropic instability, J. Atmos. Sci., 40 (1983), pp. 1029-1036.

[24] R. S. Lindzen and K. K. Tung, Wave overreflection and shear instability, J. Atmos. Sci., 35 (1978), pp. 1626-1632.

[25] M. E. McIntyre and M. Weissman, On radiating instabilities and resonant overreflection, J. Atmos. Sci., 35 (1978), pp. 11901196.

[26] J. Methven, E. Heifetz, B. J. Hoskins, and C. H. Bishop, The counter-propagating rossby-wave perspective on baroclinic instability. Part III: Primitive-equation disturbances on the sphere, Quart. J. Royal Meteor. Soc., 131, Part B (2005), pp. $1393-1424$.

[27] J. Methven, B. J. Hoskins, E. Heifetz, and C. H. Bishop, The counter-propagating rossby-wave perspective on baroclinic instability. Part IV: Nonlinear life cycles, Quart. J. Royal Meteor. Soc., 131, Part B (2005), pp. 1425-1440.

[28] W. M. Orr, Stability or instability of the steady motion of a perfect liquid, Proc. Roy. Irish Acad., 27 (1907), pp. 9-69.

[29] J. Pedlosky, Geophysical Fluid Dynamics, Springer-Verlag, New York, 2d ed., 1987. 738 pp.

[30] _ Baroclinic instability: The Charney paradigm, in The Atmossphere - A Challenge, The Science of Jule Charney, R. S. Lindzen, E. N. Lorenz, and G. W. Platzman, eds., no. 8 in American Meteorological LSociety Historicaal Monograph Series, 
American Meteoroloical Society, Boston, 1990, pp. 159-176.

[31] R. T. Pierrehumbert and K. L. Swanson, Baroclinic instability, Ann. Rev. Fluid Mech., 27 (1995), pp. 419-467.

[32] M. R. Schoeberl and R. S. Lindzen, Numerical simulation of barotropic instability. Part I: Wave-mean flow interaction, J. Atmos. Sci., 41 (1984), pp. 1368-1379.

[33] M. Takahashi, On the shear instability without over-reflection, J. Meteor. Soc. Japan, 64 (1986), pp. $793-804$.

[34] K. Takano, On the mechanism of overreflection of a barotropic Rossby wave, J. Meteor. Soc. Japan, 76 (1998), pp. $191-203$.

[35] G. K. Vallis, Atmospheric and Ocean Fluid Dynamics: Fundamentals and Large-scale Circulation, Cambridge University Press, New York, 2006. 745 pp.

[36] M. Yamada and M. Okamura, Overreflection and overtransmission of Rossby waves, J. Atmos. Sci., 41 (1984), pp. $2531-2535$. 